\section{Summary}

We have successfully used sonicated liposomes made of egg yolk phosphatidylcholine, cholesterol and stearylamine at the molar ratio $4: 3: 1$ for intravenous administration of lipophilic drugs to human beings. Three substances have been studied so far : two hydrophobic cytostatic agents, NSC 251635 a quinazolone derivative and 6-aminochrysene a polycyclic aromatic amine, and an amphiphilic antifungal agent, amphotericin B. Tolerance to liposomal therapy was excellent despite the use of large volumes. 174 infusions have been performed so far. Prolonged presence "of the drugs in the blood has been observed and, for amphotericin $B$, higher serum peak and through values have been measured as compared to its colloidal preparation, Fungizo$\mathrm{ne}^{\mathrm{R}}$. The liposomes used appeared to be preferentially taken up by the liver. We conclude that liposomes are useful drug carriers deserving further investigation in medicine.

\section{ADRESSE}

J.P. Sculier, A. Coune, C. Brassinne, C. Lauduron, C. Hollaert : service de médecine et laboratoire d'investigation clinique $\mathrm{HJ}$. Tagnon, institut Jules Bordet, centre des tumeurs de l'université libre de Bruxelles, 1, rue Héger-Bordet, 1000 Bruxelles, Belgique. $m / s n^{\circ} 1$ ool. 3, janoier 87

\title{
Premiers essais d'utilisation intraveineuse du liposome ultrasoniqué en thérapeutique humaine
}

\author{
J.P. Sculier, A. Coune, C. Brassinne, \\ C. Laduron, C. Hollaert
}

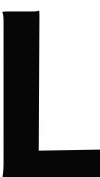

es liposomes sont des vésicules lipidiques constituées d'une ou plusieurs couches bimoléculaires de phospholipides séparées les unes des autres par un espace aqueux (figure 1, page suivante). Ces structures artificielles constituent un modèle de membrane biologique et sont susceptibles d'avoir de multiples applications, dont l'administration de médicaments [1]. En effet, le liposome peut servir de vecteur biodégradable à des substances hydrophiles occupant les compartiments aqueux, à des substances lipophiles s'incorporant dans les bicouches lipidiques ou à des composés amphiphiles* se distribuant entre ces deux phases. Il peut changer le devenir pharmacologique de ces médicaments, par exemple en modifiant leur distribution tissulaire, en augmentant leur demi-vie ou en réduisant leur toxicité. Notre recherche s'est particulièrement orientée vers l'administration intraveineuse de grands volumes de liposomes ultrasoni- qués contenant des substances hydrophobes. Le but de cet article est de présenter un résumé des résultats que nous avons déjà obtenus chez l'homme quant à la tolérance d'un tel traitement et, dans le cas de l'administration d'un antifongique, quant à son efficacité. Les protocoles thérapeutiques utilisés ont été précédemment soumis au comité d'éthique de l'institut Jules Bordet et ont été approuvés.

\section{Choix du liposome}

Notre choix résulte d'expériences in vitro [2] montrant une interaction particulière entre un liposome bien défini et la cellule leucémique expérimentale L1210. Il s'agit d'un liposome [3] ultrasoniqué constitué de phosphatidylcholine de jaune d'œuf, de cholestérol et de stéarylamine dans la proportion molaire $4: 3: 1$ et dont la concen-

- amphiphile = drogue présentant un pôle hydrophile at un pôle hydrophobe. 
tration lipidique est de 10 ou $20 \mathrm{mg} / \mathrm{ml}$. Le principe de la préparation consiste à faire éclater sous I'effet des ultrasons à haute énergie des liposomes multilamellaires contenant la substance hydrophobe pour obtenir des liposomes unilamellaires. Comme les propriétés pharmacologiques des substances incorporées dans un liposome peuvent varier suivant sa composition et sa structure, nous nous sommes tenus à l'étude d'un seul liposome de composition bien déterminée.

\section{Administration d'agents cytostatiques hydrophobes}

Le premier médicament que nous avons administré à l'aide de liposomes est un dérivé de la quinazolone, le NSC 251635 (figure 2a). Cette substance, incorporée dans le liposome, s'est révélée active contre diverses tumeurs expérimentales dont la leucémie L1210 [3]. L'étude pilote de perfusions de grands volumes de liposomes vecteurs de cet agent antinéoplasique a été réalisée chez des patients porteurs d'un cancer au-delà de toute possibilité thérapeutique conventionnelle et acceptant en toute connaissance de cause une thérapeutique expérimentale $[4,5]$. Quarante perfusions de liposomes à $20 \mathrm{mg}$ de lipides par ml, d'un volume variant de 205 à $1000 \mathrm{ml}$ ont été pratiquées chez 14 patients ; trois d'entre eux furent traités par de multiples perfusions. La tolérance au traitement a été bonne, sans complications cardiopulmonaires, ni toxicité hématologique. Aucun effet antitumoral n'a cependant été observé. Une légère sédation a été observée chez quelques patients.

Nous étudions actuellement un autre cytostatique hydrophobe, le 6 -aminochrysène (figure 2b). Il s'agit d'une amine aromatique polyclique [6] ayant montré en administration orale une certaine activité antitumorale chez la femme porteuse de cancer du sein mais s'accompagnant d'une toxicité digestive majeure qui avait fait abandonner son utilisation. Comme le taux d'incorporation de

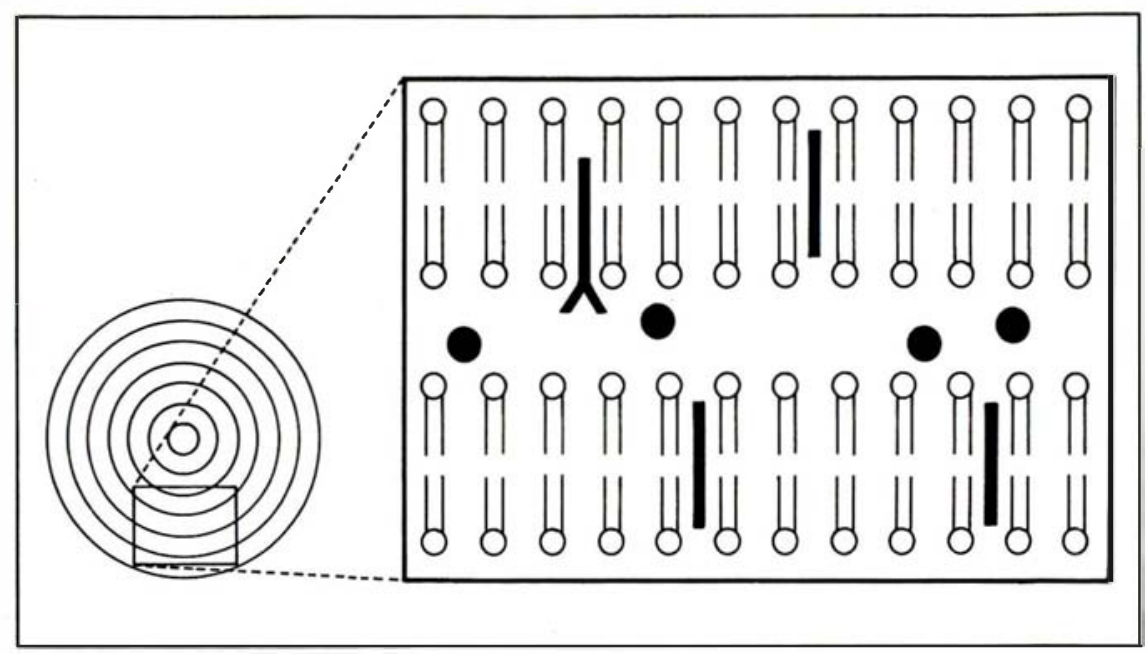

Figure 1. Représentation schématique d'un liposome multilamellaire. - composé hydrophile; - composé lipophile; $\succ$ composé amphiphile.

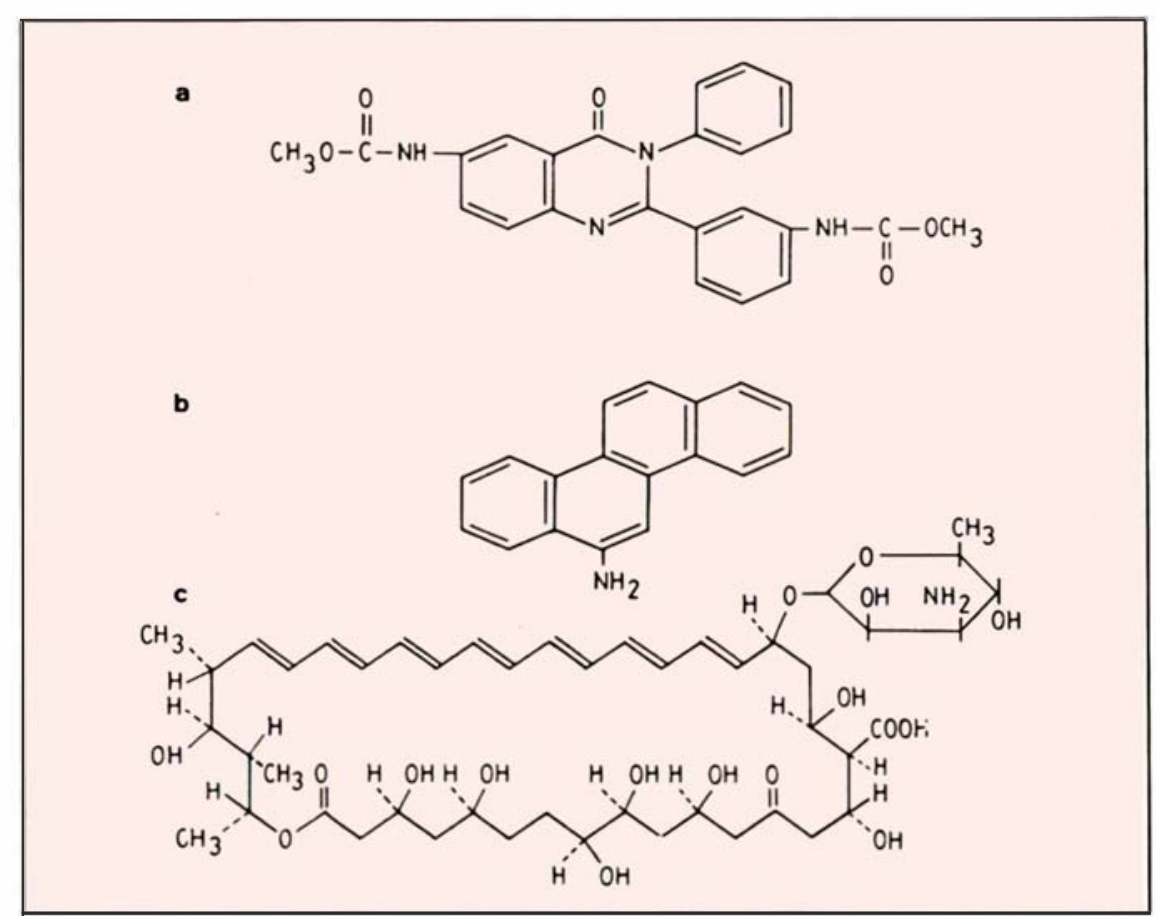

Figure 2. Formule des médicaments étudiés. a : NSC 251635 ; b : 6 aminochrysène; $c$ : amphotéricine $B$. 


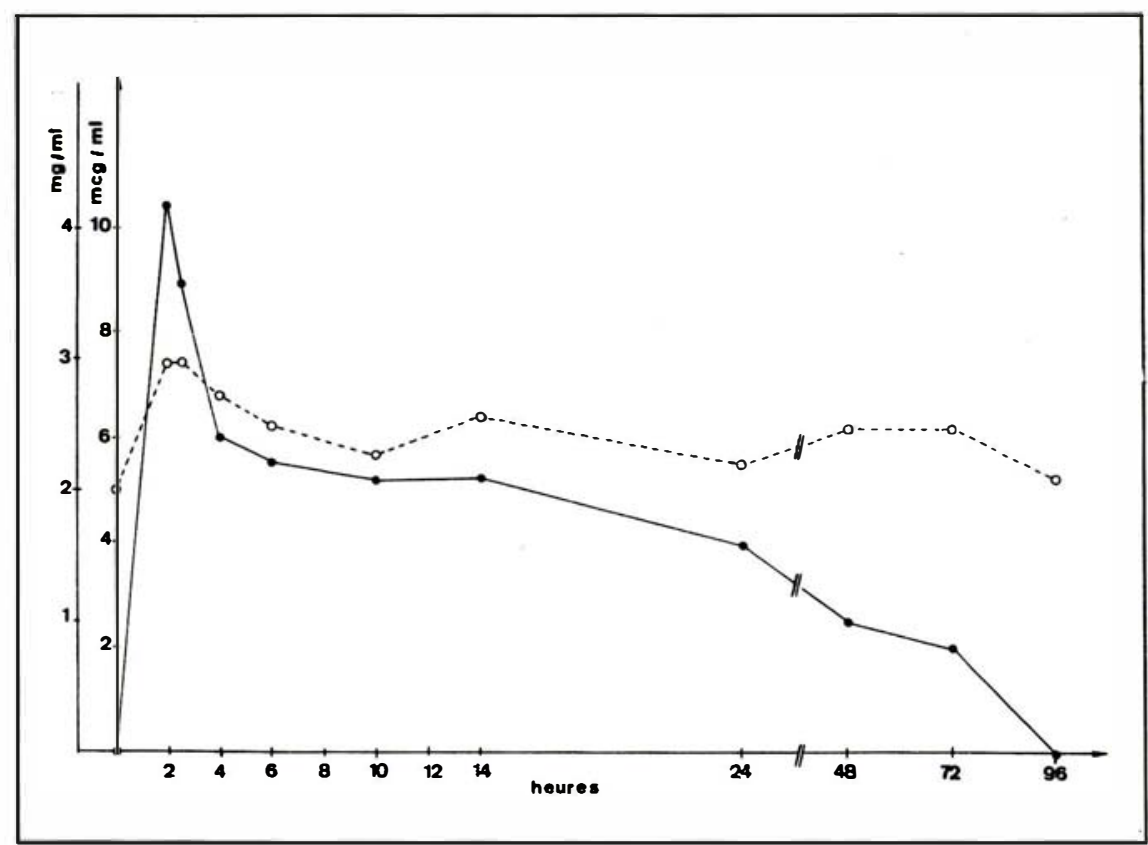

Figure 3. Pharmacocinétique de l'évolution sérique du NSC $251635(-)$ et des phospholipides (--) après perfusion en deux heures de $500 \mathrm{ml}$ de liposomes contenant $330 \mathrm{mg}$ de cytostatique. ultrasoniqués à la concentration de $10 \mathrm{mg}$ de lipides par $\mathrm{ml}$ est élevé, nous l'avons également injectée sous cette forme à des malades atteints d'un cancer avancé. Trente-quatre perfusions ont été réalisées chez neuf patients. La dose initiale de $30 \mathrm{mg} / \mathrm{m}^{2}$ de 6-aminochrysène a été progressivement augmentée pour atteindre une dose de $160 \mathrm{mg} / \mathrm{m}^{2}$ dans un volume de liposomes d'un peu moins d'un litre. La tolérance de cette préparation est également très bonne : nous n'avons observé qu'une légère sédation chez quelques patients. Une réponse objective, c'est-à-dire une réduction de plus de $50 \%$ de la surface tumorale, a été observée au niveau d'une métastase cérébrale et d'une métastase surrénalienne après 4 perfusions, pratiquées à 15 jours d'intervalle, chez un patient porteur d'un épithélioma glandulaire bronchique généralisé en récidive liposomes. L'explication de ce phénomène reste encore à établir. Au moins deux facteurs peuvent intervenir : d'une part une interaction avec les lipoprotéines plasmatiques avec "capture " d'une partie des liposomes, d'autre part une forte activation du système du complément observée aussi bien avec le NSC 251635 qu'avec le 6-aminochrysène. Celle-ci pourrait être responsable de la destruction d'une fraction des liposomes avec redistribution de ses composantes. La biodistribution des liposomes a été étudiée en les marquant à $1^{\prime 111}$ In [7] et en les injectant à des patients recevant le NSC 251635. Une captation hépatique précoce a été observée atteignant $13,5 \%$ de la radioactivité totale du corps après 20 minutes et $17 \%$ après 20 heures; dans la rate, la captation était de l'ordre de $3 \%$ pour moins de $3 \%$ dans les reins et les poumons. Il n'a pas été mesuré de captation accrue par la tumeur. Notons que la distribution tissulaire est la même, que les liposomes marqués contiennent ou non le médicament. Ces expériences suggèrent une captation préférentielle de nos vésicules par le système réticuloendothélial hépatique.

cérébrale après irradiation. Cet effet clinique, particulièrement encourageant, devra être confirmé par le traitement d'un plus grand nombre de patients.

La pharmacocinétique de la préparation contenant le NSC 251635 a été analysée [5]. La courbe des' concentrations du composé dans le sérum (figure 3) montre un pic contemporain de la perfusion suivie d'une phase de décroissance rapide puis d'une phase d'élimination très lente. $\mathrm{La}$ demi-vie de la phase précoce est de l'ordre de deux heures tandis que celle de la phase tardive approche les 24 heures. L'élévation de la concentration sérique des phospholipides est proportionnelle à la quantité de liposomes administrée. Le volume de distribution des phospholipides est beaucoup plus petit que celui du NSC 251635. Cette observation suggère une dissociation partielle mais rapide du composé et des

\section{Administration d'un antifungique amphiphile}

L'amphotéricine B est actuellement l'antifungique le plus puissant. Sa faible solubilité dans l'eau conduit à l'administrer sous forme d'une suspension colloïdale (Fungizone $^{R}$ ) obtenue grâce à l'utilisation d'un agent dispersant, le désoxycholate de sodium. L'injection intraveineuse de cette préparation entraîne fréquemment des effets indésirables tels que frissons, fièvre, céphalées, vomissements è néphrotoxicité. De plus, elle se montre peu active dans les mycoses de l'immunodéprimé.

Le taux d'incorporation de l'amphotéricine B dans nos liposomes ultrasoniqués est élevé. Cette préparation, que nous appelons ampholiposomes, montre dans des tests in vitro une importante activité antifungique. Aussi 


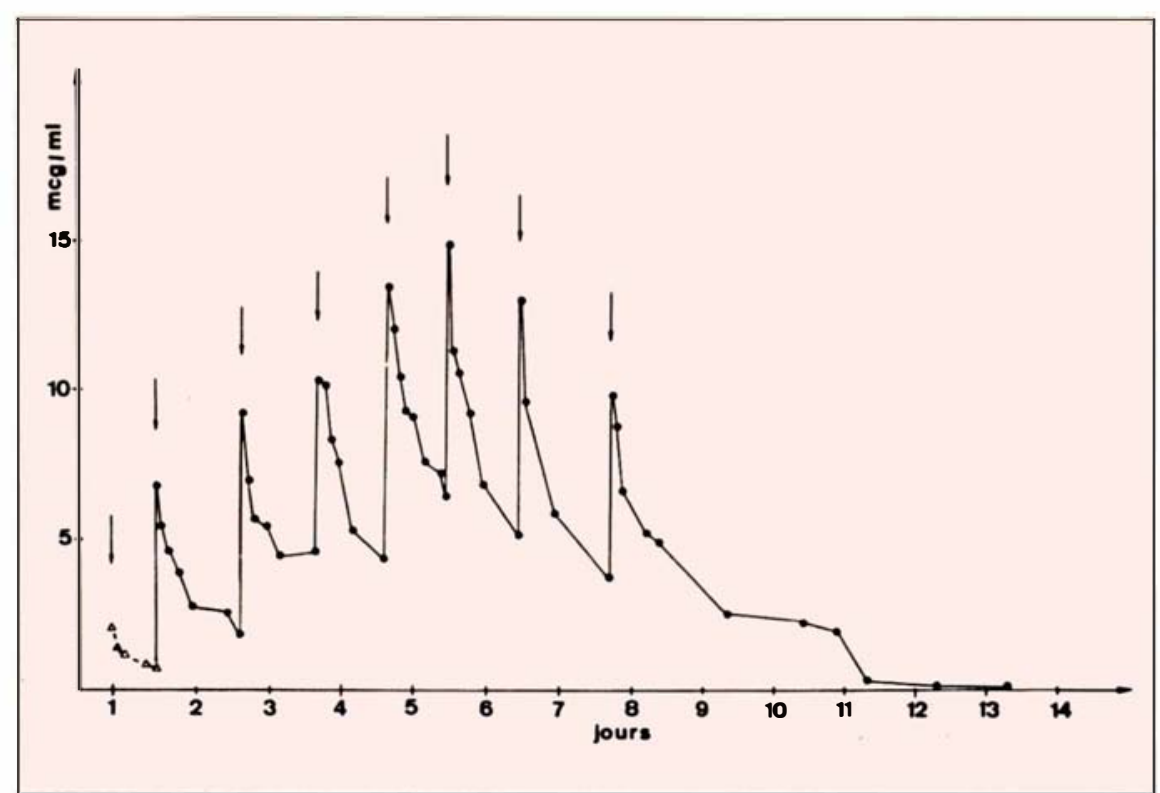

Figure 4. Pharmacocinétiquo de l'évolution sérique de l'amphotéricine $B$ chez un patient ayant reçu successivement de la Fungizone ${ }^{R}(-\Delta-\Delta-)$ et des ampholiposomes (-).

avons-nous débuté une étude pilote chez des patients cancéreux souffrant de mycoses systémiques graves. Un total de 100 perfusions a été réalisé. Douze patients ont reçu, avec une remarquable tolérance, de une à 20 fois les ampholiposomes, la dose d'amphotéricine $\mathrm{B}$ étant le plus souvent comprise entre 300 et $600 \mathrm{mg}$. La tolérance rénale a non seulement été excellente mais encore, chez les malades ayant une insuffisance rénale secondaire à l'administration préalable de Fungizone ${ }^{R}$, celle-ci s'est améliorée durant le traitement par les ampholiposomes. Chez la plupart des malades, l'administration antérieure de Fungizone ${ }^{R}$ avait été en fait très mal supportée. Bien que le but principal de l'étude ne fût pas de déterminer l'activité antifungique chez le malade, plusieurs aspergilloses pulmonaires sévères ont répondu de façon assez remarquable aux ampholiposomes malgré un contexte de neutropénie. De plus, une très nette aug- d'amphotéricine $\mathrm{B}$ a été mesurée (figure 4). Alors que les pics sériques ne dépassent guère $2 \mu \mathrm{g} / \mathrm{ml}$ avec la Fungizone ${ }^{\mathrm{R}}$, ils atteignent aisément 10 à $20 \mu \mathrm{g} / \mathrm{ml}$ avec les ampholiposomes, les vallées se situant entre 5 et $10 \mu \mathrm{g} / \mathrm{ml}$. Les liposomes permettent donc d'accroître l'index thérapeutique de l'amphothéricine $B$, non seulement en améliorant nettement sa tolérance mais vraisemblablement en augmentant son activité antifungique.

\section{Conclusion}

Nos investigations ont permis de montrer qu'un liposome ultrasoniqué de composition bien déterminée peut servir de vecteur pour l'injection intraveineuse à l'homme de substances hydrophobes, sans induire de toxicité importante. Ce mode d'administration permettra dans un avenir proche d'élargir l'arsenal thérapeutique à un groupe de composés négligés jusqu'à présent

\section{RÉFÉRENCES}

1. Coune A, Sculier JP, Brassinne C, Laduron C. Les perspectives d'avenir du liposome en thérapeutique. Rev Med Brux 1983; 4 : 617-22.

2. Frühling J, Penasse W, Laurent G, et al. Intracellular penetration of liposomes containing a water insoluble antimitotic drug in L1210 cells. Eur J Cancer 1980 ; 16 : 1409-16.

3. Brassinne C, Atassi G, Frühling J, et al. Antitumor activity of a water-insoluble compound entrapped in liposomes on L1210 leukemia in mice. $J$ Natl Cancer Inst 1983; 70 : 1081-6.

4. Coune A, Sculier JP, Frühling J, et al. IV administration of a water-insoluble antimitotic compound entrapped in liposomes. Preliminary report on infusion of large volumes of liposomes to man. Carcer Treat Rep 1983 ; 67 : 1031-3.

5. Sculier JP, Coune A, Brassinne C, et al. Intravenous infusion of high doses of liposomes containing NSC 251635, a water insoluble cytostatic agent. A pilot study with pharmacokinetic data. J Clin Oncol $1986 ; 4$ : 789-97.

6. Groupe Européen du cancer du sein. Induction par le 6-aminochrysène de rémission du cancer du sein en phase avancée chez la femme. Eur J Cancer 1967 ; 3 : 75-7.

7. Frühling J, Coune A, Ghanem G, et al. Distribution in man of ${ }^{1 l 1}$ In-labelled liposomes containing a water-insoluble antimitotic agent. Nuclear Med Communications 1984 ; 5 : 205-8.

\section{TIRÉS A PART}

J.P. Sculier, A. Coune, C. Brassinne, C. Laduron, C. Hollaert : service de médecine et laboratoire d'investigation clinique $\mathrm{HJ}$. Tagnon, institut Jules Bordet, centre des tumeurs, de l'université libre de Bruxelles, 1, rue Héger-Bordet, 1000 Bruxelles, Belgique. 